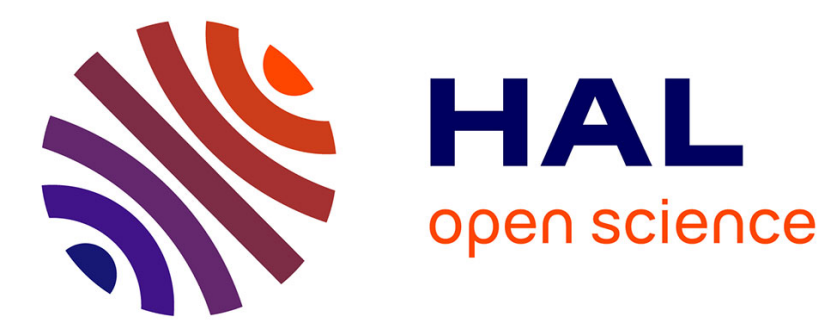

\title{
Les stratégies du vignoble oléronais à l'épreuve de la transformation du territoire insulaire et de la dépendance au bassin de production charentais : se diversifier, résister et se distinguer
}

Bedrani Naila, Alban Landré

\section{To cite this version:}

Bedrani Naila, Alban Landré. Les stratégies du vignoble oléronais à l'épreuve de la transformation du territoire insulaire et de la dépendance au bassin de production charentais : se diversifier, résister et se distinguer. Norois, 2020, 2 (255), pp.37-55. 10.4000/norois.9772 . hal-03162364

HAL Id: hal-03162364

https://hal-univ-rennes1.archives-ouvertes.fr/hal-03162364

Submitted on 8 Mar 2021

HAL is a multi-disciplinary open access archive for the deposit and dissemination of scientific research documents, whether they are published or not. The documents may come from teaching and research institutions in France or abroad, or from public or private research centers.
L'archive ouverte pluridisciplinaire HAL, est destinée au dépôt et à la diffusion de documents scientifiques de niveau recherche, publiés ou non, émanant des établissements d'enseignement et de recherche français ou étrangers, des laboratoires publics ou privés. 
Les stratégies du vignoble oléronais à l'épreuve de la transformation du territoire

2 insulaire et de la dépendance au bassin de production charentais : se diversifier, résister et se distinguer

4 Titre en Anglais

The strategies of the Oleron vineyards at the test of the transformation of the island's territory

6 and dependence on the Charente production area : diversification, resistance and distinction

8 Titre court pour les pages pour les pages paires

Les stratégies du vignoble oléronais

10 Auteurs

Naïla Bedrani a* et Alban Landré b

12 a ESO-Rennes, UMR CNRS 6590, Université Rennes 2, Place du recteur Henri Le Moal, CS 24307, 35043 Rennes Cedex, France (naila.bedrani@univ-rennes2.fr). Auteur correspondant ; 14 tel : +33 (0)2 99141000 .

b Arènes, UMR CNRS 6051, Université de Rennes 1, Faculté de droit et de science politique,

169 rue Jean Macé - CS 54203, 35042 Rennes Cedex

*Auteur correspondant : Tel : +33(0)2 99141000

18

Résumé 
20 La viticulture joue un rôle fondamental pour l'île d'Oléron, tant d'un point de vue socioéconomique que paysager, et ce depuis au moins la fin du Moyen Âge. La filière viticole

22 oléronaise s’inscrit dans le bassin de production du Cognac, en périphérie ouest de l’AOC. En situation de marginalité par rapport aux crus les plus prestigieux de l'appellation, soumis aux

24 aléas d'un marché tourné vers l'exportation, le vignoble a occupé sur l'île une superficie très fluctuante : de 6000 hectares dans la première moitié du XIXe siècle, soit alors pratiquement

26 un tiers du territoire oléronais, à 750 hectares au début des années 2010. Après la déstabilisation due au phylloxera autour de 1880, la viticulture oléronaise a dû composer avec le rapide

28 développement du tourisme sur l'île et à la nouvelle concurrence qu'il impliquait sur le foncier dans la seconde moitié du XXe siècle. Elle a également été confrontée aux politiques

30 d'arrachage pour réguler la production. Face à ces obstacles, les acteurs de la filière ont tenté d'adapter leurs modèles économiques et de protéger leur accès à la terre. Nous analysons ces

32 dynamiques de résistance au prisme de l'approche évolutionniste développée en géographie économique. Le processus d'apprentissage et d'innovation qui a marqué la filière viticole

34 oléronaise s'est traduit par une mise à profit des ressources territoriales disponibles, dans le but d’atténuer la dépendance de l'île au bassin de production du Cognac. La coopération avec les

36 élus locaux, la diversification des produits et des marchés, la montée en gamme et la mise en avant d'un terroir singulier au sein de l'appellation, ont participé d’une (re)territorialisation de

38 la viticulture et semblent aujourd'hui contribuer à la relative stabilité foncière et économique de la filière.

40

Abstract

42 Viticulture has been essential for Oléron island, both from a socio-economic and landscape point of view, since at least the end of the Middle Ages. Oléron winegrowing sector is part of

44 Cognac production basin and situated on the western fringes of the Protected Geographical Indication (PGI). This peripherical position compared to the most prestigious vintages of the

46 PGI, and the hazards of a heavily export-oriented market, meant that Oléron wine growing lands get through large fluctuations : from 6,000 hectares in $19^{\text {th }}$ century, which amouts to one third

48 of the total surface of the island, to 750 hectares in 2010. During the second half of the $20^{\text {th }}$ century, Oléron winegrowing sector had found an additional difficulty in the rapid growth of

50 tourism, which provoked a tougher competition for land. It was confronted as well to production limitations through the uprooting of premiums. Facing these obstacles, stakeholders from 
52 Oléron winemaking sector have carried out economical adaptation efforts and protection of their access to land. We analyse their reaction in light of the evolutionist approach developed

54 by economical geographers. Innovations mobilized through a learning process has seemed to allow the Oléron winegrowing stakeholders to soften the path dependency to the Cognac

56 production basin capitalizing on the available territorial resources. Cooperation with local representatives, product and market diversification, upmarketing, and the spotlight put on the

58 specificity of this terroir have contributed (re)territorialise winegrowing at the island level. This strategy seems to be bringing some stability to land property and better economical outputs to 60 the sector.

62 Mots clés

Vignoble, filière, innovation, île, littoral Atlantique, reterritorialisation, Oléron

64 Keywords

Vineyards, sector, innovation, island, Atlantic coastline, reterritorialisation, Oléron

66 Article reçu le 7 octobre 2019 ; définitivement accepté le 5 mai 2020.

Suivi éditorial : Céline Barthon

\section{INTRODUCTION}

Les premiers questionnements sur la viabilité économique des activités agricoles dans les îles

70 de la façade atlantique française émergent dans la seconde moitié du $\mathrm{XX}^{\mathrm{e}}$ siècle (Dumont, 1951 ; Dumortier, 1976) alors que l'agriculture française s'inscrit dans un élan de modernisation sans

72 précédent. L'agriculture française se transforme radicalement, à la fois sur les plans sociotechniques, politiques et économiques, pour répondre aux rôles de productivité puis de

74 compétitivité à l'export qui lui sont assignés. La polyculture-élevage traditionnelle laisse place à une spécialisation des systèmes agricoles et donne naissance aux bassins de production

76 comme « espaces spécialisés dans un petit nombre de spéculations et polarisés par les industries d'amont et d'aval, les services, le grand commerce » (Rieutort, 2009). Le terme de « dé-

78 territorialisation » de l'agriculture est employé pour qualifier la transformation d'un modèle agricole et alimentaire local par un modèle agroalimentaire industriel mondialisé qui échappe

80 en grande partie aux agriculteurs (Rieutort, 2009). L’insularité comme marginalité géographique (Brigand et al., 2019) génère à la même période des conditions défavorables à 
82 l'intégration de l'agriculture insulaire dans ces nouvelles filières agroalimentaires et entrave ainsi la pérennité des exploitations agricoles autrefois orientées majoritairement vers la

84 production vivrière (Brigand, 2002).

Pourtant, dès le Moyen Âge, les îles de Noirmoutier, Ré et Oléron s’inscrivent dans une

86 économie spéculative autour de la production de sel et de vin, en complémentarité avec la production auto-consommée (Guillemet, 1999). L’île d’Oléron profite comme la plupart des

88 îles d'une position géographique d'avant-poste (Brigand et al., 2019) dans ce cas favorable aux activités commerciales. Les seigneurs oléronais tirent profit de cette situation jusqu'aux crises

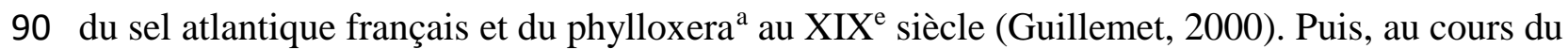
$\mathrm{XX}^{\mathrm{e}}$ siècle, la paysannerie viticole oléronaise devenue propriétaire doit affronter les défis du

92 bassin de production du Cognac fortement dépendant d'un marché mondialisé. Dès la première moitié du XX $\mathrm{X}^{\mathrm{e}}$ siècle, la viticulture oléronaise doit également composer avec le développement

94 du tourisme qui engendre des mutations dans l'organisation spatiale de l'île. Ces mutations s’accroissent avec l'ouverture du pont en 1966. Le foncier insulaire faiblement régulé

96 s’artificialise au détriment d'une agriculture peu rémunératrice par rapport aux perspectives offertes par le marché foncier à vocation résidentielle et de loisirs. Les espaces agricoles

98 reculent au profit de l'enfrichement et d'une urbanisation rétro littorale diffuse, tandis que s’intensifie le développement des activités de loisirs sur le cordon littoral de l'île (Barthon,

100 2002). Sur nombre de littoraux et d'îles, les surfaces agricoles et le nombre d'agriculteurs se réduisent et « le monde agricole littoral [...] ne peut survivre que s’il oppose un dynamisme

102 fort et une réelle originalité » (Lebahy et Le Délézir, 2007). Cette attractivité résidentielle et touristique des territoires ruraux favorisent l'émergence de nouveaux marchés (Hervieu et

104 Viard, 1996). La vente directe et les circuits-courts sont alors une opportunité commerciale pour nombre d'exploitations exclues de la modernisation agricole. Par ailleurs, le développement de

106 labels de type AOC et IGP principalement, permet aux producteurs engagés dans ces démarches collectives de se démarquer sur les circuits longs, par la valorisation de la qualité et de la

108 spécificité des produits commercialisés (Chiffoleau, 2019). Dynamique d’ancrage renouvelé, la (re)territorialisation de l’agriculture s’opère alors autour de trois leviers que sont la durabilité,

110 l'origine des produits et la patrimonialisation et « reflète ainsi un sentiment identitaire tout en créant de la sociabilité et de la solidarité. Pour les acteurs du développement, elle désigne à la

112 fois la structuration organisée d’une petite région, de ses acteurs et de ses projets, selon des

a Nom à la fois d'un insecte ravageur de la vigne et de la maladie qu'il transmet aux racines du cep de vigne. 
formes variables, et la manière dont les politiques publiques sont en mesure de s’adapter à ce 114 processus » (Rieutort, 2009).

Dans cette perspective, nous souhaitons interroger la dynamique empiriquement observée de 116 (re)territorialisation de la filière viticole oléronaise qui semble désormais tirer parti de son ancrage territorial, tant géographique que socio-économique, malgré les différentes pressions

118 ayant remis en cause sa pérennité tout au long du $\mathrm{XX}^{\mathrm{e}}$ siècle.

\section{MÉTHODE}

Nous inscrivons notre démarche dans une perspective disciplinaire de géographie économique

122 et dans la dynamique des travaux portant sur le développement territorial et les proximités. Analyser par la focale territoriale, les processus économiques et leurs transformations dans un

124 monde globalisé, permet de rendre compte d'innovations, de mutations, de processus d’adaptation et de résilience, au sein des espaces ruraux notamment (Pecqueur, 2014 ; Pecqueur

126 et Peyrache-Gadeau, 2010). En effet, l'ancrage spatial des acteurs, les formes de gouvernance territoriale et la construction sociale de l'espace vécu interfèrent dans les processus de

128 production et de consommation, même si ces derniers s'inscrivent dans le modèle économique capitaliste et libéral dominant. L’approche retenue est centrée sur l’identification de processus

130 complexes multi-échelons et de relations multi-acteurs, ancrés spatialement, historiquement et culturellement, et n’incluant pas uniquement des opérateurs économiques liés au marché. En

132 termes de méthode, «l'approche par le territoire, [le corpus] se fonde d'abord sur les organisations productives localisées et leurs capacités à s’autoréguler en lien avec des structures

134 sociales et institutionnelles et à évoluer sous l'effet de perturbations exogènes ou d'innovations endogènes. [...] Les résultats portent sur la spécificité des réponses, sur la singularité des

136 trajectoires et des modes de gouvernance » (Pecqueur et Peyrache-Gadeau, 2010).

Nous faisons ici l’hypothèse que la persistance de la filière viticole oléronaise, en périphérie de

138 la région de production du Cognac et aux prises avec le développement du tourisme depuis le début du $\mathrm{XX}^{\mathrm{e}}$ siècle, est le résultat de multiples mutations portées par un système d'acteurs

140 évolutif qui s'ancre progressivement dans l'espace socio-économique, politique et géographique qu'est le territoire oléronais, lui-même en transformation. Pour opérer cette

142 territorialisation de la production viticole, nous supposons que les acteurs de la filière ont fait preuve d'innovations entendues comme « toute nouveauté qui provoque un changement dans

144 les modes de fonctionnement antérieurs » (Torre, 2015). Tel que défini dans la théorie en 
construction du développement territorial proposée par Torre, nous mobilisons la définition des

146 innovations territoriales qui «font appel à l'inventivité des populations locales, sans être obligatoirement liées à un fort niveau d'industrialisation ou de spécialisation productive »,

148 caractérisées la plupart du temps par une dimension coopérative et « par leur capacité à recréer des proximités et maintenir des solidarités locales». Ces innovations peuvent être «soit

150 produites par différentes composantes du tissu économique et social, en réponse à des impacts exogènes ou endogènes ou des initiatives locales, soit importées et imitées d'expériences

152 menées ailleurs. » (Torre, 2018). Ces innovations sont des processus et elles peuvent être technologique, organisationnelle, sociale ou encore institutionnelle. Il s'agit alors de les

154 caractériser, de les restituer dans le temps long du territoire et de s’intéresser à leurs conditions d'émergence et de gouvernance selon des dynamiques qui peuvent être coopératives et

156 concertatives ou conflictuelles et concurrentielles. Pour ce faire, nous nous inspirons de l'approche évolutionniste en tenant compte des contingences du passé pour contribuer à

158 expliciter la situation contemporaine. Ainsi, nous proposons ${ }^{\mathrm{b}}$ une première caractérisation des étapes clés de la trajectoire de la filière viticole oléronaise en nous appuyant sur une

160 démonstration historique et narrative afin de caractériser les innovations qui ont provoqué « des bifurcations, des mutations et des modifications de trajectoires, qui initient de nouveaux

162 chemins de développement » (Torre, 2015).

Pour appréhender la trajectoire de la filière viticole oléronaise, nous avons consulté la littérature

164 grise mise à disposition par les acteurs locaux (DREAL, SAFER, Communauté de communes) ${ }^{\mathrm{c}}$. Nous avons réalisé des entretiens semi-directifs avec une viticultrice (âgée de plus de 50 ans, 166 en viticulture biologique depuis huit ans, sur une ferme en polyculture), un viticulteur (âgé de plus de 60 ans, sur une ferme conventionnelle exclusivement viticole mais qui a eu un atelier 168 maraîchage dans les années 1970) et un retraité de la viticulture (ancien viticulteur, toujours

\footnotetext{
b Ce travail s'inscrit dans le cadre du projet de recherche pluridisciplinaire SOFIANE qui vise à analyser les caractéristiques structurelles, sociales, environnementales, économiques et politiques des agricultures des îles de la façade atlantique française. D’un point de vue méthodologique, cette étude de cas est l'objet d'une approche inductive et s'inscrit plus largement dans un travail d'enquêtes exploratoires. Elle alimente le projet en complémentarité de travaux en cours sur l'analyse des facteurs d'insularité dans les filières alimentaires des îles de la façade atlantique française et sur la gouvernance agricole et alimentaire de ces territoires.

c Nous avons consulté des documents d'urbanisme (schémas de cohérence territoriale de 1989 et 2005) ainsi que des documents de la Direction Régionale de l’Environnement de l’Aménagement et du Logement (Exposition « Oléron fragile, regards sur les transformations récentes des paysages oléronais », 2005 ; Cahier des charges pour un rapport d'expertise sur le phénomène de camping sur parcelles privées, 2016) de la Société d’Aménagement Foncier et d'Établissement Rural (étude de l'enfrichement de l'île d'Oléron, 2002) et de la Communauté de communes de l'île d'Oléron (Grille des prix du foncier agricole, 2011 ; Charte de l'agriculture durable de l'île d’Oléron, 2012 ; Diagnostic agricole, 2015).
} 
actif sur la ferme familiale, âgé de plus de 80 ans). Ces trois viticulteurs ont tous eu des

170 responsabilités professionnelles au cours de leurs carrières (coopératives, syndicats, SAFER, associations de développement agricole locales, mais pas de mandat d’élu local). Nous avons

172 également réalisé des entretiens avec des salariés des coopératives agricole (1) et viticole (1) de l'île, des agents (2), un élu (1) et un ancien élu (1) de la Communauté de communes ${ }^{\mathrm{d}}$ de l'île

174 d'Oléron. Ces entretiens s'inscrivaient dans une phase d'enquêtes exploratoires plus large sur l’agriculture de l’île (14 personnes interrogées). Le rôle historique et structurant de la viticulture

176 a été abordé par chaque enquêté (sans préméditation de la part des enquêteurs) et a permis de discuter des évolutions de cette filière emblématique dans le territoire oléronais. Par ailleurs,

178 nous avons fait appel à la statistique agricole mise à disposition par le Bureau National Interprofessionnel du Cognac pour qualifier les transformations structurelles du vignoble

180 oléronais au cours du temps et au sein de son bassin de production.

Dans un premier temps, nous présenterons les principales difficultés rencontrées par la filière

182 viticole oléronaise au cours du $\mathrm{XX}^{\mathrm{e}}$ siècle. Dans un deuxième temps, nous présenterons les bifurcations recherchées et les innovations mises en œuvre, à partir des années 1960. Dans un

184 troisième temps, nous nous intéresserons plus particulièrement à la patrimonialisation du vignoble comme levier de développement socio-économique.

\section{LA PÉRENNITÉ DU VIGNOBLE MENACÉE}

\section{En marge de l’AOC Cognac}

Depuis le milieu du Moyen Âge, les producteurs de l'île d’Oléron, à la fois paysans, sauniers

190 et viticulteurs étaient intégrés dans des filières d'exportation (Guillemet, 1999). La demande en vin dès le XIII ${ }^{\mathrm{e}}$ siècle, puis en eaux-de-vie à partir du XVII ${ }^{\mathrm{e}}$ siècle, s'amplifie au sein d'un

192 marché international $^{\mathrm{e}}$ et favorise le développement du vignoble depuis la côte atlantique vers l'intérieur des terres en remontant le fleuve Charente (figure 1).

d Ancien élu pour le mandat 2008-2014 ayant contribué au lancement du programme d'actions intercommunautaire de maintien et développement de l'agriculture durable de l'île d'Oléron. Élu actuel pour le mandat 2014-2020, participant à la poursuite du programme d’actions agricole.

e Guillemet (1999) évoque « une demande économique en sel et vin liée aux circuits nationaux et internationaux » qui s’accroît « à partir du XIIIe siècle, avec les besoins des villes du Nord et surtout de la pêche, en particulier au hareng puis à la morue ». Denieau (1994) précise qu'Oléron passe sous l'influence de la couronne d'Angleterre au $\mathrm{XII}^{\circ}$ siècle, suite au mariage d'Aliénor d'Aquitaine et Henri Plantagenêt devenu roi d'Angleterre, permettant ainsi le développement du commerce de vins avec l’Europe du Nord. Bernard (1988) souligne qu' «en 1860, la 
194 Ce commerce fait dès lors vivre une économie viticole régionale spécialisée dont font partie les îles de Ré et d’Oléron en périphérie ouest (Bernard, 1988). De 1872 à 1890, le phylloxéra touche

196 fortement le vignoble des Charentes (figure 2) qui perd 85 \% de sa surface. Les îles sont un peu moins touchées car nombre de plantations localisées dans des sols sablonneux ou inondés

198 l’hiver ont été épargnées par ce ravageur peu friand de ces milieux (Denieau, 1994). Sur l'île d’Oléron, un quart des vignes subsiste (figure 3) et les replantations de cépages destinés à la

200 distillation font qu'au début du $\mathrm{XX}^{\mathrm{e}}$ siècle le vignoble insulaire s'étend sur un peu plus de 4000 hectares (Denieau, 1994). A contrario, de nombreuses surfaces en vigne sur le continent et

202 notamment sur le littoral sont reconverties dans l'élevage laitier et la production de céréales. La zone de production de Cognac se recentre autour de la ville de Cognac (Bernard, 1980). Dans

204 ce contexte de forte diminution spatiale du vignoble, des risques de fraude sont craints face au décalage entre demande toujours croissante en eaux-de-vie et production alors restreinte. La

206 concurrence d'autres alcools est aussi redoutée (Bernard, 1980). La mise en place d'une législation spécifique a alors pour objectif la protection des eaux-de-vie charentaises. Le décret

208 de 1909 fixe les délimitations géographiques de l'aire de production. Le décret d’appellation Cognac de 1936 détermine les procédés et celui de 1938 entérine les crus ou sous-appellations

210 régionales. Ce classement tient compte des conditions pédoclimatiques et des qualités organoleptiques des eaux-de-vie obtenues et détermine six crus. A une exception près, les aires

212 des crus s’inscrivent dans une architecture concentrique avec les terres qualifiées de Grande Champagne au centre de l’AOC jusqu'aux terres classées en Bois Ordinaires, incluant les îles

214 de Ré et d'Oléron (figure 4). "En périphérie de l’AOC, les Bois Ordinaires ou 'Bons Bois”' produisent des eaux-de-vie aux arômes fruités qui vieillissent rapidement » (Bernard, 1980). Ce

216 gradient défavorable aux îles se traduit par des prix de vente moins rémunérateurs que ceux pratiqués au centre de l’AOC. Une coupure prend forme entre le Cognaçais et les îles qui

218 s’éloignent du centre commercial établi autour des sous-appellations les plus prestigieuses. Les aléas du marché du Cognac, par la fluctuation des prix et de la demande mondiale rendent donc

220 les producteurs oléronais relativement plus vulnérables que leurs collègues du centre de l’appellation Cognac.

signature du traité de libre-échange fait de l’Angleterre un grand marché de redistribution vers l'Europe du Nord et les colonies ». 


\section{La dépendance au bassin de production du Cognac}

De 4000 hectares de vignes au début du XX $X^{\mathrm{e}}$ siècle, l'île d'Oléron en compte environ 1800 à la

224 sortie de la Seconde Guerre Mondiale. Les deux grandes guerres qui provoquent un manque de main-d’œuvre pour entretenir le vignoble, ainsi que la dépendance aux grandes maisons de

226 négoce pour la commercialisation, sont les principales causes de cette perte de surface. Entre 1925 et 1956, à l’initiative des viticulteurs oléronais, cinq coopératives de vinification et de

228 distillation sont créées dans l'île afin d’obtenir plus de poids face aux négociants (figure 3). Le développement du mouvement coopératif à cette période permet de ne pas voir le vignoble

230 totalement disparaître (Bernard, 2016).

Dans les années 1950 et 1960, le marché du Cognac connaît un essor remarquable.

232 L’accroissement de la demande entraîne l’ouverture de droits de plantation pour les cépages destinés à la distillation. Alors que les acteurs de la viticulture oléronaise souffrent

234 économiquement de leur marginalisation au sein de l'AOC, ils tentent de tirer avantage de ce contexte favorable, d'autant plus que cet essor est concomitant à la modernisation des outils de

236 production. Les exploitations s’agrandissent, se modernisent et se spécialisent comme en témoigne un viticulteur retraité : «De 5 hectares, on est passé à 15 hectares grâce aux droits

238 de plantations qui avaient été attribués par le Bureau National du Cognac. On était dans une période faste pour le Cognac. Les négociants disaient: on va manquer de Cognac, il faut

240 planter ». Un autre rapporte ses souvenirs du propos qu’il attribue au député de l'époque « Plantez des vignes, c'est un devoir national », tant le Cognac vendu majoritairement à

242 l'exportation était important dans la balance commerciale française (figure 5). Ainsi, des années 1960 aux années 1970, le vignoble dans l’aire de production du Cognac passe de 64000 hectares

244 à 110000 hectares (figure 2). Sur Oléron, cet engouement se traduit par une progression contenue des surfaces viticoles, de l'ordre de 200 à 400 nouveaux hectares, du fait du

246 morcellement du foncier et de la concurrence pour la terre avec l'urbanisation et les loisirs (Bernard, 2016 ; Denieau, 1994). En 1972, le vignoble oléronais recouvre entre 2000 et 2200

248 hectares, superficie qui représente à cette époque $30 \%$ des Bois Ordinaires et 2,5\% de l'ensemble de l'appellation Cognac (figure 3).

250 Suite à cette période faste, trois phases de crises s'enchaînent. Après cette période d'expansion spatiale du vignoble, la première crise brutale démarre en 1973 face à une situation de 252 surproduction au cœur d'une conjoncture commerciale peu favorable : en 1975, environ 75\% 
de la production de Cognac est exportée à l'étranger ${ }^{f}$ (Bernard, 1978) et les stocks d'eau-de-vie

254 représentent sept années de récolte alors que les ventes diminuent de 20\% (Bernard, 1988). Les revenus des viticulteurs sont extrêmement dépendants des politiques commerciales et de

256 régulation de la consommation d'alcool menées par les États importateurs, ainsi que des stratégies de stockage et de mise en marché de négociants peu nombreux ${ }^{g}$, qui disposent d'un

258 fort pouvoir sur la filière. Cette première crise est suivie d'une deuxième au début des années 1980 puis d'une troisième au début des années 1990. Pour faire face à ces crises de

260 surproduction, une prime à l'arrachage est instaurée en 1980. Le recours à cette prime atteint son apogée en 1987 avec 17000 hectares arrachés dans l’année dans le bassin de production du

262 Cognac (Bernard, 1988) : «On en a tellement planté qu'en 1975, on a vu le début d'une grande crise qui a duré on va dire jusqu'en 1988, un truc comme ça. Et on a dû arracher... on avait

264 tellement bien planté qu'on a eu une prime d'arrachage. Il s'est arraché dans les Charentes quelque chose comme 20000 hectares. Je dis peut-être des bêtises, mais en gros tout ce qu'on

266 avait planté on l'a arraché” (viticulteur, avril 2019). Le dispositif de la prime à l'arrachage est davantage mobilisé dans les sous-appellations périphériques que dans les sous-appellations

268 centrales (figure 6). Le vignoble de l'île d’Oléron perd environ 1000 hectares en une décennie, soit la moitié de sa surface viticole (figure 7). La viabilité des outils coopératifs insulaires est

270 mise en péril du fait de la baisse des apports des coopérateurs.

La filière oléronaise est ainsi fortement marquée par les aléas de la filière Cognac dans son

272 ensemble et par sa position en marge de l'AOC. Par ailleurs, la filière connaît également des pressions singulières en relation avec le développement du tourisme dans l’île.

\section{En marge d'une destination touristique en plein essor}

A partir des années 1960, l'essor du tourisme entraîne le développement de parcelles à camper

276 privées, de constructions résidentielles, l’aménagement de campings et de colonies de vacances. Ce phénomène est favorisé par l’ouverture en 1966 du pont reliant Oléron au continent. Les

278 campeurs et résidents secondaires se rendent facilement sur l'île, y compris avec une caravane.

f A cette époque, 150 pays importent du Cognac. Les six premiers pays consommateurs en 1976 sont les USA, le Royaume-Uni, l'Allemagne, les Pays-Bas, Hong-Kong et la Belgique-Luxembourg. 60 \% des volumes sont exportés au sein de la Communauté Économique Européenne. Les volumes exportés par pays sont très aléatoires d'une année à l'autre mais le niveau d'exportation est relativement stable puisqu'il était déjà de 76 \% en 1956 (Bernard, 1978). Depuis les années 1990, les exportations représentent environ $95 \%$ à $98 \%$ des volumes commercialisés. Depuis 2008, le podium des pays importateurs est composé des États-Unis en tête, suivis par Singapour et la Chine (BNIC, 2019).

g En 1976, quatre entreprises de négoce réalisent 70 \% des ventes (Bernard, 1978). Le vieillissement des eaux-devie, le stockage et la mise en marché est une étape majoritairement effectuée par ces maisons de négoce. 
Cette consommation de foncier agricole pour l'urbanisation et les loisirs, et le mitage qui en

280 découle, se déploient dans un contexte caractérisé par un micro-parcellaire dense, issu de la nécessité historique pour les familles de disposer d'une variété de terrains suffisante pour

282 produire ce dont elles avaient besoin au quotidien. Les divisions successives lors des partages des héritages ont également participé à la complexification de la situation foncière dans les îles

284 (Péron, 1993 ; Guillemet, 2000). Comme en témoigne une viticultrice, « on s’est retrouvés avec des timbres-poste à travailler pendant une centaine d'années », jusqu'aux remembrements qui

286 ont eu lieu dans trois communes sur huit, des années 1960 aux années 2000.

En pleine période de modernisation agricole, les techniques viticoles évoluent très vite et le

288 vignoble se restructure avec l'arrivée des tracteurs enjambeurs puis de la machine à vendanger.

Diviser les terres agricoles et les vendre en tant que parcelles à camper ou à construire permet

290 ainsi aux agriculteurs oléronais d'augmenter leurs revenus et/ou d'investir dans du matériel agricole (Sanson, 2008). Les viticulteurs et les propriétaires se séparent principalement des

292 terres les moins adaptées aux nouvelles modalités de culture et de récolte et les valorisent au prix du terrain constructible ou de loisirs. Parallèlement aux agriculteurs et viticulteurs qui

294 vendent quelques terrains dans l'objectif de faciliter la modernisation de leur outil de production, d'autres exploitations sont complètement démantelées par leurs propriétaires

296 retraités, leurs héritiers ou des acheteurs extérieurs. Deux problèmes sont alors simultanément résolus pour les cédants : la reprise des terres est assurée (même si la transaction détourne le

298 bien de sa vocation agricole) et la retraite est financièrement confortée en procurant un apport complémentaire aux pensions agricoles. Ce phénomène est observable sur nombre de littoraux

300 à partir des années 1960 (Lebahy et Le Délézir, 2007). L’ampleur des conversions de terres agricoles vers ces autres usages a été facilitée par une non-régulation du marché foncier par les

302 politiques publiques ${ }^{\mathrm{h}}$ : «Les vendeurs c'étaient des électeurs, des gens qui restaient dans l'île. Et donc si jamais les maires s'étaient mis en travers de cette politique-là, ils allaient se mettre

304 à dos la plupart de ceux qui voulaient vendre » (viticulteur retraité, avril 2019). Près de 8000 parcelles à camper sur Oléron sont dénombrées à la fin des années 1970 (DREAL). Encore

306 aujourd'hui, de nombreux propriétaires conservent des surfaces anciennement agricoles dans l'espoir qu'elles soient rendues constructibles, ce qui provoque par la rétention foncière, un

308 enfrichement estimé à 2000 hectares par une étude SAFER en 2002. La vente pour d'autres

h Cela n'est pas un trait spécifique à l'île d’Oléron ou aux îles de l'atlantique mais une caractéristique commune aux littoraux français, au moins jusqu’à l’entrée en vigueur de la « Loi Littoral » en 1986. 
usages et la rétention foncière participe avec les crises successives du marché du Cognac à la

310 diminution spatiale des espaces agricoles et du vignoble (figure 3).

\section{ENTRE RESISTANCES ET ADAPTATIONS}

Face à ces mutations, les viticulteurs les plus engagés dans la modernisation agricole et les plus

314 attachés au métier de viticulteur, vont déployer différentes innovations pour tenter de contenir la diminution des surfaces viticoles et de maintenir la viabilité des outils de production, 316 notamment en enrôlant d'autres acteurs locaux, qu'ils soient collègues, administratifs ou élus.

\section{Protection foncière et opposition à la prime à l'arrachage}

318 Dans le contexte d'expansion du tourisme à partir des années 1950, une partie des acteurs de la filière viticole oléronaise se retrouve partie prenante de conflits d'usage, entendus comme une

320 « forme de résistance et d'expression des oppositions d'une partie de la population locale à des décisions des pouvoirs publics ou d’opérateurs privés qui la laissent insatisfaite » (Torre et al.,

322 2016). Leur mobilisation vise à faire face à la remise en cause de la vocation productive des espaces agricole engendrée par la perte de surfaces, le mitage et l'ébranlement du marché

324 foncier agricole. Suite à la mise en service du pont en 1966 et jusqu'au milieu des années 1970, l’urbanisation se développe considérablement et « la spéculation foncière bat son plein dans

326 l'indifférence générale » (Nadreau, 1994). En 1975, un projet de camping est envisagé sur trois hectares de terres agricoles situées au milieu d'une zone viticole sur la commune de Saint-Pierre

328 d’Oléron. Des agriculteurs ${ }^{\mathrm{i}}$ manifestent pour obtenir l'abandon du projet. Le syndicat départemental FDSEA appuie les démarches de la section locale, notamment en apportant son

330 soutien dans le financement des procédures judiciaires pour le maintien de la vocation agricole des terrains. Les viticulteurs interrogés expriment l'absence d’anticipation de la majorité des

332 élus locaux de l'époque pour faire face à ces menaces, faute d'attribuer un avenir à la filière : « les agriculteurs on était bons à jeter. La viticulture fonctionnait mal. Il fallait absolument faire

334 des campings partout » alors qu'en l'absence de POS $^{\mathrm{j}}$, la SAFER ${ }^{\mathrm{k}}$ ne peut intervenir. Cet événement s'ensuit d'une mobilisation des responsables professionnels de l'île pour réclamer

336 la formalisation de POS dont le premier de l'île est approuvé en 1977 et le dernier en 1994. Une

\footnotetext{
i Environ 200 agriculteurs manifestants selon les enquêtés.

j Les Plans d'Occupation des Sols sont des documents d'urbanisme instaurés par la loi d'orientation foncière de 1967 qui déterminent les servitudes d'utilisation des sols (dont la possible interdiction de construire).

k Société d’Aménagement Foncier et d’Équipement Rural. En Région Poitou-Charentes, elle est instaurée en 1962 en même temps que l'État confère aux SAFER le droit de préemption.
} 
fois établis, ces POS permettent de contenir l'urbanisation mais ne règlent pas le problème de 338 la pratique illégale du camping sur parcelles privées en zone agricole et naturelle. Néanmoins, la SAFER peut dès lors jouer son rôle pour s'opposer aux transactions qui entraîneraient un

340 changement de vocation des biens agricoles. Un viticulteur oléronais était alors président de la SAFER départementale et régionale : «Il a entraîné, on l’a suivi, il a entraîné derrière lui une

342 majorité d'agriculteurs pour protéger les terres de l'île » (viticulteur, avril 2019). Pour autant, l'espoir que les terrains deviennent constructibles demeure et participe au phénomène de

344 rétention foncière encore aujourd'hui.

Ces mobilisations pour assurer une protection foncière des espaces agricoles via les politiques

346 d’urbanisme se conjuguent à la mobilisation pour supprimer la prime à l'arrachage instaurée en 19791. «La prime à l'arrachage avait inquiété un certain nombre de viticulteurs parce que les

348 anciens agriculteurs qui eux n'avaient plus l'espoir, plus d'enfants [pour reprendre après eux], ne savaient pas trop quoi en faire. Ils ont sauté sur l'occasion pour toucher la prime. Résultat,

350 ça faisait des friches entre deux vignes car ils avaient arraché et ils n'exploitaient pas derrière. Ça nous a fortement inquiétés car on s'est dit: les vignes vont disparaître d'Oléron »

352 (viticulteur retraité, avril 2019). La mise en œuvre de cette politique d'arrachage fait à nouveau réagir les responsables professionnels : «On a fait une action jusqu’à Bruxelles pour exonérer

354 Ré et Oléron de la prime d'arrachage pour sauver le vignoble de Ré et d'Oléron, pour faire en sorte qu'il y ait une certaine surface viticole qui reste » (viticulteur retraité, avril 2019). Ils

356 sollicitent d'abord la Chambre d'agriculture de la Charente Maritime puis le Ministère de l'Agriculture et seront enfin orientés ensuite vers la Direction Générale Agriculture de la

358 Communauté Économique Européenne. L’opposition à ce dispositif public de régulation de la production est rapportée comme « une réaction de viticulteurs dynamiques qui croyaient dans

360 l'avenir pour faire exempter les îles de la prime d'arrachage » bien qu'il ne fasse pas l’unanimité parmi les viticulteurs (GEDAR, Marennes Oléron TV, 2015). La prime est

362 supprimée en 1989 après avoir contribué à la disparition de la moitié du vignoble oléronais. Conjointement à ces mobilisations collectives en interaction avec les institutions, des

364 démarches d'adaptation sont entreprises au cœur même des outils de production et vont également modifier l’organisation de la filière.

l Directive européenne 79/359/CEE du Conseil du 26 mars 1979, relative au programme d'accélération de la reconversion de certains vignobles dans la région des Charentes. 


\section{Le maraîchage pour réduire la vulnérabilité des exploitations viticoles}

A partir de 1975, le développement des productions maraîchères dans les exploitations viticoles

368 oléronaises, permet de pallier en partie les difficultés économiques liées au marché du Cognac. Sous l'impulsion du Groupement de Développement Agricole de l'île d'Oléron et de son

370 conseiller, une quarantaine de viticulteurs se lancent dans la culture des légumes pour répondre à la demande locale saisonnière : "Le premier été en 1980 [après la diversification de

372 l'exploitation viticole vers le maraîchage], je suis allée au marché de Domino avec ma petite 4L. J'avais de l'ail et des haricots verts, une petite table de deux mètres avec trois paniers, une

374 balance Robert Val et on a fait notre première saison comme ça et en fait d'années en années, on a développé, on s'est mis à planter des tomates, on a cultivé des patates, après des melons.

376 Ça a été progressif» (viticultrice, avril 2019). Cette bifurcation dans les trajectoires des exploitations se fait progressivement, en limitant les investissements aux tunnels, petits

378 équipements relatifs à la vente et dispositifs d'arrosage, sans recours important à l'emprunt. Les légumes demandent peu de surface et se valorisent bien par la vente directe, notamment auprès

380 des estivants : «On avait la chance d'être dans l'île d'Oléron où tu ouvres ta porte et les clients sont là. [...] à ce moment-là il y avait deux mois de saison donc tu allais au marché l'été, tu

382 vendais tes légumes et puis voilà : ça mettait du beurre dans les épinards » (viticultrice, avril 2019). La plupart des viticulteurs qui développent des ateliers ${ }^{\mathrm{m}}$ maraîchage conservent leurs

384 parcelles avec l'espoir d'une reprise des marchés du Cognac: «A l'époque, j'avais 5 à 6 hectares de vignes en propriété, plus du fermage. Les gars [propriétaires des parcelles mises

386 en fermage] ont arraché leurs vignes, on a gardé nos 5 hectares [en propriété] seulement. [...] On a gardé les vignes mais on allait tailler dans les vignes pour des poires » (viticulteur, avril

388 2019).

Dans un premier temps très défavorable au maintien spatial de l'agriculture, le développement

390 de la vocation touristique d'Oléron offre de nouveaux débouchés rémunérateurs dont se saisissent les viticulteurs de l'île par la production de légumes commercialisés en vente directe.

392 En 2015, sur les quarante-deux exploitations viticoles de l'île, treize ont encore plusieurs productions dont six un atelier maraîchage. Les ateliers maraîchage dans les fermes viticoles

394 ont progressivement été supprimés à la suite de la remontée des cours du Cognac.

m Une exploitation agricole peut rassembler plusieurs productions (viticulture, maraîchage, élevage, grandes cultures...) dont les conduites sont différenciées tout en étant inclues dans le fonctionnement global de l'exploitation. Nous parlons donc d'ateliers pour distinguer ces productions et leurs organisations propres au sein d'une même entité juridique et productive. 


\section{La diversification des produits viticoles, une bifurcation vers de nouveaux}

396 marchés

Dès les années 1980, les viticulteurs indépendants de l'île sont précurseurs de nouvelles 398 orientations productives pour répondre au marché saisonnier émergent en lien avec le tourisme. Longtemps cantonné à une production marginale pour la consommation familiale dans le bassin

400 de production du Cognac, le Pineau est de plus en plus élaboré pour une commercialisation locale. Composé de Cognac à $25 \%$ et de moûts issus de cépages à vin de consommation à $75 \%$,

402 c'est à la fois un nouveau débouché pour la production d'eau-de-vie oléronaise et une amorce à la modification de l'encépagement vers les cépages à vin de consommation rouge et blanc.

404 Constatant que les touristes consomment des vins issus d'autres vignobles pour accompagner la dégustation des huîtres d'Oléron (essentiellement en provenance du Muscadet), ces cépages

406 à vin de consommation deviennent également valorisables pour la production de Vins de Pays ${ }^{\mathrm{n}}$. Tandis que la production de vin pour la distillation se caractérise par de forts rendements et un

408 faible degré d'alcool, c'est le contraire pour les vins tranquilles. Les viticulteurs apprennent alors à cultiver la vigne, de la taille à la vendange, pour obtenir des jus qualitatifs ainsi qu'à

410 conduire des vinifications abouties au regard des attentes des consommateurs estivants. Les coopératives viticoles créées dans l'entre-deux-guerres ont progressivement fusionné jusqu'à

412 ce qu'il n'en reste plus qu'une en 1993. Cette dernière s'engage elle aussi dans cette diversification à partir de 1995. Afin d'atténuer la dépendance au marché du Cognac, les acteurs

414 de la filière viticole oléronaise recherchent encore aujourd'hui un équilibre économique entre leurs trois produits viticoles : «Chez moi, j'ai du chardonnay. J'ai des merlots, des cabernets,

416 du sauvignon, tout ça c'est pour faire du vin qu'on vend l'été [aux touristes]. On a une filière Pineau, c'est important pour nous et nos collègues indépendants, ça reste le produit phare pour

418 l'île d'Oléron, et on a une filière Cognac qui est encore assez intéressante » (viticulteur, avril 2019).

420 Finalement, en 2018, les surfaces en vigne occupent 85000 hectares dans la région délimitée Cognac dont seulement 1576 hectares en sous-appellation Bois Ordinaires. Le vignoble

422 d'Oléron, avec 805 hectares, concentre 51\% des surfaces de cette sous-appellation (contre 30\% en 1972) et l'île de Ré, 36\%. A elles seules, les deux îles représentent donc 87 \% de la sous-

n Le cépage principal pour produire le Cognac est l'ugni blanc. L’orientation de la production vers le pineau blanc et rosé et les vins tranquilles se traduit par la plantation d'autres cépages : chardonnay, sauvignon, merlot, cabernet franc, pinot noir en sont les principaux. 
424 appellation Bois Ordinaires. Le cépage pour la production de Cognac ne disparaît pas de l’encépagement oléronais mais il se cantonne à 65\% de la surface du vignoble insulaire contre

426 93\% à l'échelle de la région délimitée Cognac alors que 20\% de la surface est consacrée à la production de Vins de Pays contre 2\% à l'échelle régionale. A titre d'illustration, 30\% des

428 ventes de la coopérative viticole oléronaise (tous produits confondus) se fait en direct dans le magasin, au siège à Saint-Pierre d'Oléron ${ }^{0}$, alors qu'à l'échelle de l'AOC, l'export de Cognac

430 concerne $98 \%$ des volumes produits.

Les innovations que constituent les diversifications temporaires ou permanentes (maraîchage,

432 diversification des produits viticoles) et les résistances aux projets d'aménagements locaux et aux mesures de restructuration du bassin de production ont façonné des liens entre la filière

434 viticole et son environnement institutionnel local qui facilitent le développement progressif d’instruments pérennes d'action publique, au croisement de dynamiques de développement 436 territorial.

\section{La maîtrise foncière}

438 La mise en tourisme des îles à pont provoque des transformations paysagères et une pression sur les milieux naturels insulaires (Barthon, 2002). Les collectivités locales se saisissent alors

440 peu à peu d'outils de protection pour contenir l'urbanisation diffuse et le camping sur parcelles privées et intègrent dans leur préoccupation la lutte contre la banalisation des paysages par

442 l'enfrichement.

Succède au Schéma Directeur de l’île approuvé en 1989, le Schéma de Cohérence Territoriale

444 du Pays Marennes-Oléron approuvé en 2005. Le maintien des activités primaires est présenté comme un objectif dans les deux documents (Sanson, 2008). En 2002, la SAFER réalise une

446 étude et une cartographie des friches sur le territoire oléronais. Bien que les collectivités locales expriment une volonté de contenir le phénomène, cette étude ne sera pas suivie d'actions

448 spécifiques. Pour autant, les viticulteurs et les agriculteurs, au travers de leurs représentants professionnels, continuent de solliciter les élus pour une meilleure prise en compte de leurs

450 problématiques, principalement foncières. Par ailleurs, les divers zonages de protection des paysages et des milieux, s’ils freinent l'urbanisation, répondent avant tout à une logique de 452 patrimonialisation (Barthon, 2005). Les politiques d'acquisition des collectivités et obtenu d'information sur la répartition des ventes par marché et par produit pour les indépendants. 
établissements publics (Espaces Naturels Sensibles du Conseil Général et acquisitions du

454 Conservatoire du littoral) qui en découlent se concentrent sur les espaces boisés, les marais et les zones agricoles mitées et marquées par la pratique du camping sur parcelle privée. Ces

456 politiques n’impliquent pas nécessairement une remise à disposition des terrains pour l'agriculture bien que certaines productions soient considérées comme des outils de gestion

458 compatibles avec des objectifs de restauration et de conservation, tels que la saliculture et élevage, mais pas la viticulture. En 2010, le service de développement économique

460 nouvellement créé à la Communauté de communes d'Oléron, intègre le développement d'une « agriculture durable » dans ses missions. Un groupe de travail sur l'accès au foncier et au bâti

462 agricole est mis en place. La Communauté de communes, les agriculteurs et la SAFER déterminent entre autres une grille indicative des prix du foncier agricole, qui reçoit

464 l'approbation de France Domaine. La Communauté de communes passe une convention avec la SAFER pour réaliser une veille foncière et intervenir par la préemption. Un budget

466 communautaire annuel est alloué à la constitution de réserves foncières agricoles. Les objectifs de cette politique foncière agricole sont finalement assez semblables à ceux des viticulteurs

468 impliqués dans les années 1970 et 1980 : enrayer la diminution de la surface agricole utile, lutter contre le mitage, limiter l'envolée des prix du foncier agricole, faciliter l'installation dans

470 un contexte nouveau où les installations se font de plus en plus hors cadre familial. Par ailleurs, en 2010, la Société Civile d’Exploitation Agricole (SCEA) Dyonis est créée par les adhérents

472 de la coopérative viticole oléronaise. Pour garantir la viabilité économique de la coopérative, cette société achète ou prend à bail et entretient les vignes de coopérateurs en fin de carrière,

474 dans l'attente de trouver des porteurs de projet à installer ${ }^{\mathrm{P}}$. Par le portage foncier et l'exploitation temporaire, l'objectif est de limiter le démantèlement des outils de production des

476 coopérateurs par le maintien d'unités viables, le temps de trouver preneur pendant une période tampon qui s’allonge du fait d'installations hors cadre familial de plus en plus nombreuses.

478 Complémentaires, ces initiatives foncières et d'aménagement esquissent un agencement d'appuis mutuels entre la viticulture, l'agriculture et les autres dynamiques économiques 480 soutenues par les collectivités locales, répondant à plusieurs objectifs que nous allons maintenant expliciter.

p Depuis 10 ans, la SCEA a acheté ou pris à bail le parcellaire de six exploitations (pour cause principale de départ en retraite) et a permis ainsi l'installation de deux personnes hors cadre familial et le renforcement de trois coopérateurs déjà en place. 


\section{LA SINGULARITÉ DU VIGNOBLE INSULAIRE COMME LEVIER D’ATTRACTIVITE ÉCONOMIQUE}

484 Depuis les années 1990, la profession s’inscrit dans une démarche de différenciation et de montée en gamme de ses produits. La valorisation porte essentiellement sur la dimension

486 culturelle de cette activité historiquement pratiquée dans l'île. Depuis les années 2010, la filière poursuit ce travail de transformation de la valeur socio-économique et identitaire attribuée cette

488 activité, avec Communauté de communes et les acteurs du tourisme. Le processus de patrimonialisation en cours est la traduction d'une dynamique collective d'appropriation,

490 caractérisée par plusieurs étapes qui peuvent être définies selon enchaînement suivant : la sélection de l'objet qui devient patrimoine, la justification, la conservation, la mise en

492 exposition et éventuellement la valorisation marchande (François et al, 2006). Il vise dans notre étude de cas, tant à renouveler le regard sur cette activité des touristes déjà friands de produits

494 locaux, que celui de la population insulaire et des acteurs du tourisme. Nous allons expliciter ce processus dans cette dernière partie.

\section{Se distinguer par l'affirmation du lien au territoire}

L’Indication Géographique Protégée “Charentais” pour les Vins de Pays rouge, rosé et blanc

498 voit le jour dans sa première version en 1981. Le cahier des charges de l’IGP autorise le complément de la mention “Charentais" par une précision géographique ciblée qui

500 correspondant à cinq secteurs différents : Ile de Ré, Saint-Sornin, Ile d’Oléron, Charente, Charente-Maritime. Les dénominations Saint-Sornin et Ile de Ré sont obtenues en 1992 et la

502 dénomination Ile d’Oléron en 1999. «Les vins de pays [...] sans apporter le prestige d’une Appellation d'Origine Contrôlée fournissent toutefois des garanties d'origine et d'élaboration

504 et apportent surtout l'authenticité d'un territoire qui seul permet la composition d'un fonds oenoculturel » (Maby, 2002). Pour les îles, à rebours du classement périphérique dans l’AOC

506 Cognac, cette mention supplémentaire autorisée souligne au début des années 2000 la notoriété émergente de ces deux vignobles, porteurs pour l'ensemble de la filière. En ce sens, le cahier

508 des charges ${ }^{q}$ précise que les vins de l’IGP se caractérisent par « des profils sensoriels et aromatiques facilement appréhendés par les consommateurs et recherchés, notamment par la

510 clientèle touristique. Aujourd'hui, la commercialisation des vins en IGP Charentais est particulièrement active au plan régional et bénéficie de l’attrait touristique de la zone côtière en

q Cahier des charges de l’Indication Géographique Protégée “Charentais” homologué par arrêté du 26 octobre 2011 et modifié par arrêté du 24 juillet 2015. 
512 période estivale ». L’AOC Pineau des Charentes, officialisée par décret en 1945, n’autorise pas de mention relative à un espace géographique plus restreint. Pour autant, les acteurs de la filière

514 viticole réunis au sein du syndicat des Vins de Pays Charentais île d’Oléron, associés à leurs homologues de l'île de Ré, travaillent depuis 2012 avec le Comité National du Pineau des

516 Charentes pour obtenir la déclinaison d’une mention géographique, aujourd'hui toujours en instruction. Par ailleurs, des viticulteurs de Ré et d’Oléron, ainsi que des maisons de négoce,

518 mentionnent déjà l’origine géographique insulaire pour caractériser des Pineaux et Cognacs qu’ils commercialisent, mention légalement interdite puisque ces produits sont protégés par

520 l’AOC dont le périmètre est bien plus large.

Afin d'ancrer un peu plus la viticulture oléronaise dans son histoire et d'en affirmer les

522 particularités, le Groupement d'étude et de développement agricole et rural $^{\mathrm{r}}$ met en œuvre différents outils de promotion. Ses bénévoles organisent de 1989 à 2000 des circuits à vélo en

524 saison, « Deux roues pour une île », pour faire découvrir les activités primaires, le patrimoine bâti, culturel et naturel de l'île. Les fermes viticoles font partie des étapes incontournables. Plus

526 récemment le GEDAR a co-réalisé un documentaire avec la télévision du Pays MarennesOléron sur l’histoire de la viticulture oléronaise ${ }^{\mathrm{s}}$. Ce phénomène de construction et publicisation

528 d'un héritage ne se cantonne pas aux espaces touristiques comme l'île d’Oléron. Les dynamiques de distinction par l'usage de l'origine géographique se retrouvent également dans

530 d'autres cas de vignobles périphériques de grandes zones d’appellation. On peut mentionner l’exemple du Pays Foyen ${ }^{t}$, dont les vins ont été longtemps commercialisés sous la seule

532 appellation “Bordeaux” alors qu'une AOC “Sainte-Foy-Bordeaux” existe depuis 1937. L’appellation est réutilisée à partir de la fin des années 1990 pour se distinguer des autres vins

534 de Bordeaux (Crenn et Téchoueyres, 2005). Cette dynamique de différenciation et d’ancrage culturel de la viticulture oléronaise se conjugue à d'autres stratégies d'attractivité touristique et

536 de durabilité à l’œuvre sur le territoire.

r Ex-groupement agricole féminin de l’île d’Oléron. Les GEDAR sont créés dans les années 1960 et animés par les MSA (Mutualité Sociale Agricole). Celui d’Oléron était composé entre autres d'agricultrices et d'ostréicultrices et avait pour vocations principales la montée en compétence collective de ces adhérentes et le lien social. Il existe toujours et s'est ouvert aux hommes. Il a aujourd'hui vocation à faire du lien entre producteurs, élus et citoyens consommateurs.

s Vin'Ile Histoire De La Viticulture oléronaise / Du Phylloxera A Nos Jours, « Ce film vous fera découvrir en image, pourquoi et comment l'histoire du vignoble oléronais joue et a joué un rôle important pour le territoire, tant d'un point de vue économique et culturel que paysager. » https://tube.mo-tv.fr/cat/2015/video/vin-ile-histoire-dela-viticulture-oléronaise-du-phylloxera-a-nos-jours

t A la limite entre le Bordelais et la Dordogne, mais dans la zone d’appellation Bordeaux. 


\section{Entre préoccupations environnementales et marketing territorial}

538 La diversification des produits viticoles oléronais s’est accompagnée d’une montée en gamme de la filière par la restructuration du vignoble et l'apprentissage de nouveaux processus de

540 production pour répondre à un marché local et régional principalement lié au tourisme. L’origine géographique est mobilisée comme levier de création de valeur ajoutée en écho à ce

542 marché. Par cette rencontre entre le producteur et le consommateur, nos enquêtés soulignent que les qualités organoleptiques et l'impact environnemental de la production sont devenus

544 sujet de réflexion. La santé au travail pour les agriculteurs comme pour leurs salariés est aussi mentionnée comme source de questionnements. Dans une démarche d'amélioration des

546 pratiques, certains viticulteurs ont exprimé la volonté de réduire l'usage d'insecticides. Pour ce faire, en partenariat avec la coopérative agricole d'approvisionnement de l'île et la

548 Communauté de communes, la confusion sexuelle (pratique alternative aux insecticides utilisés contre le ver de la grappe) est ainsi expérimentée depuis 3 ans et va concerner 200 hectares en

550 2020, soit 25\% du vignoble oléronais. Par ailleurs, la première conversion à la viticulture biologique sur Oléron a été engagée en 2010. En 2018, l’île comptait sept exploitations viticoles

552 certifiées en agriculture biologique ou en conversion pour une surface totale de 133 hectares ${ }^{\mathrm{u}}$ soit 16,5 \% du vignoble insulaire, ce qui est bien plus qu’à l'échelle du bassin de production du

554 Cognac dont les surfaces viticoles certifiées ne dépassent pas les 2 \%v En 2019, un groupe d’échanges et de suivi dédié à la viticulture biologique est créé sur l'île.

556 Alors que la reconnaissance de la qualité et des terroirs procure une plus grande résistance économique aux vignobles (Péres, 2009), ce processus s’accompagne souvent d'une mise en

558 avant du vignoble et de la viticulture en tant que paysage et savoir-faire à valoriser, tel un patrimoine historique à vocation touristique (Yengué et Robert, 2018) mis en exergue par la

560 construction d'un discours collectif et d'actions coordonnées de promotion. Un viticulteur exprime le caractère pittoresque des images qui peuvent être véhiculées : «dans le virage de la

562 piste cyclable si vous regardez le clocher et que vous prenez une photo, vous avez la même à St-Emilion ». A ceci près, que les vignes oléronaises s’imbriquent dans un paysage et une offre

564 touristique profondément marqués par la présence du littoral et de l’océan. Ce contexte en fait

u Source : Agence Bio, Les données brutes de certification AB par EPCI au 31/12/2018. https://www.agencebio.org/vos-outils/les-chiffres-cles/

v A titre de comparaison, en 2016, les surfaces viticoles certifiées biologique ou en conversion représentent 11\% du vignoble français. Ce chiffre masque de fortes disparités régionales : les départements de Charente et CharenteMaritime où se déploie l'AOC Cognac se caractérisent par une surface en viticulture biologique comprise entre 0 et $2 \%$ de leur surface viticole totale (Source Agence bio, 2017). 
son originalité par rapport à d'autres vignobles mais explique également les profondes 566 similitudes de trajectoires avec le vignoble rétais. Néanmoins, cela participe d'un mouvement assez général, selon Jacques Maby, de promotion commerciale du vin par ses paysages

568 typiques, réels ou mythifiés (2002). En ce sens, la destination "Vignoble de l’île d’Oléron” construite localement est labellisée Vignobles et Découvertes depuis 2014. La Communauté de

570 communes a été le chef d'orchestre de ce travail partenarial préalable : "Vignobles et Découvertes, c'est un label d'une destination oenotouristique, donc on travaille avec la

572 coopérative mais aussi des indépendants, ça permet de fédérer aussi certains viticulteurs entre eux et de mettre en valeur la production locale et puis d'avoir une entrée pour travailler sur

574 d'autres projets comme les pratiques culturales” (chargée de mission, Communauté de communes de l'île d’Oléron, avril 2019). Cette initiative récente de formalisation d'une offre

576 oenotouristique, qui était jusque-là inexistante, peut être interprétée comme un axe de développement complémentaire au tourisme à vocation balnéaire. Outil de marketing territorial,

578 ce projet est également pensé pour accompagner les entreprises dans une démarche de développement durable de leurs pratiques viti-vinicoles. Dans une perspective d'apaisement

580 des relations entre professionnels et collectivités et de coopération, un élu rencontré affirme que la mise en place d'une mission de maintien et de développement des activités agricoles au sein

582 de la Communauté de communes est liée à une volonté de « retour aux sources », voire même de «réparer l'histoire » au regard des évolutions foncières et économiques des activités

584 agricoles insulaires liées à la mise en tourisme de l'île. Il s’agit de reconstruire une image et un discours positifs sur les activités primaires et leur rôle pour leur territoire auprès des élus, de la

586 population locale et des acteurs du tourisme (hébergeurs, restaurateurs...) qui en sont potentiellement les principaux vecteurs. De plus, et bien qu’Oléron jouisse d’une démographie

588 hivernale importante, l'engouement actuel des élus locaux pour l'agriculture pourrait participer d'un mouvement plus global de soutien aux activités économiques à l'année et pas seulement 590 sur la période estivale.

\section{CONCLUSION}

592 Nous avons cherché à rendre compte (tableau 1) des étapes clés de la trajectoire de la viticulture oléronaise aux prises avec les fluctuations du marché du Cognac et le développement du

594 tourisme.

\begin{tabular}{|l|l|l|l|}
\hline $\begin{array}{l}\text { Objectifs de } \\
\text { l'innovation }\end{array}$ & Moyens mobilisés & $\begin{array}{l}\text { Nature et } \\
\text { dynamique } \\
\text { d'innovation }\end{array}$ & $\begin{array}{l}\text { Système d'acteurs } \\
\text { associé et gouvernance }\end{array}$ \\
\hline
\end{tabular}




\begin{tabular}{|c|c|c|c|}
\hline \multirow[t]{3}{*}{$\begin{array}{l}\text { Sécuriser la } \\
\text { vocation des } \\
\text { espaces non } \\
\text { urbanisés et } \\
\text { enrayer la } \\
\text { diminution des } \\
\text { surfaces viticoles }\end{array}$} & $\begin{array}{l}\text { Manifestation et procédure } \\
\text { judiciaire contre des } \\
\text { projets privés } \\
\text { d'artificialisation des sols } \\
\text { soutenus par les } \\
\text { collectivités locales }\end{array}$ & $\begin{array}{l}\text { Innovation sociale } \\
\text { et institutionnelle } \\
\text { Processus } \\
\text { conflictuel }\end{array}$ & $\begin{array}{l}\text { Coordination sectorielle } \\
\text { locale et supra territoriale } \\
\text { en partenariat avec les } \\
\text { syndicats et les } \\
\text { organismes } \\
\text { professionnels du } \\
\text { département et du bassin } \\
\text { de production }\end{array}$ \\
\hline & $\begin{array}{l}\text { Sollicitation des élus } \\
\text { locaux pour la mise en } \\
\text { place de POS }\end{array}$ & $\begin{array}{l}\text { Innovation sociale } \\
\text { et institutionnelle } \\
\text { Processus } \\
\text { conflictuel }\end{array}$ & $\begin{array}{l}\text { Coordination sectorielle } \\
\text { locale des responsables } \\
\text { professionnels }\end{array}$ \\
\hline & $\begin{array}{l}\text { Lobbying auprès du } \\
\text { Ministère de l'Agriculture } \\
\text { et de la Direction Générale } \\
\text { de l'Agriculture de la CEE } \\
\text { pour supprimer la prime à } \\
\text { l'arrachage }\end{array}$ & $\begin{array}{l}\text { Innovation sociale } \\
\text { et institutionnelle } \\
\text { Processus } \\
\text { conflictuel }\end{array}$ & $\begin{array}{l}\text { Coordination sectorielle } \\
\text { locale des responsables } \\
\text { professionnels et supra } \\
\text { territoriale en partenariat } \\
\text { avec les syndicats et les } \\
\text { organismes } \\
\text { professionnels du } \\
\text { département et du bassin } \\
\text { de production }\end{array}$ \\
\hline \multirow{2}{*}{$\begin{array}{l}\text { Diversifier les } \\
\text { revenus des } \\
\text { exploitations } \\
\text { viticoles face aux } \\
\text { crises } \\
\text { successives du } \\
\text { marché du } \\
\text { Cognac }\end{array}$} & $\begin{array}{l}\text { Développement des } \\
\text { ateliers de maraîchage } \\
\text { vente directe dans les } \\
\text { fermes viticoles }\end{array}$ & $\begin{array}{l}\text { Innovation } \\
\text { technologique et } \\
\text { organisationnelle } \\
\text { Processus } \\
\text { coopératif }\end{array}$ & $\begin{array}{l}\text { Coordination sectorielle } \\
\text { locale pilotée par } \\
\text { l’animateur du GDA }\end{array}$ \\
\hline & $\begin{array}{l}\text { Diversification de } \\
\text { l'encépagement vers les } \\
\text { cépages à vin de } \\
\text { consommation (Pineau, } \\
\text { Vins de Pays), évolution } \\
\text { des techniques de } \\
\text { production viti-vinicoles et } \\
\text { développement de la vente } \\
\text { en circuits-courts }\end{array}$ & $\begin{array}{l}\text { Innovation } \\
\text { technologique et } \\
\text { organisationnelle } \\
\text { Processus } \\
\text { coopératif }\end{array}$ & $\begin{array}{l}\text { Coordination sectorielle } \\
\text { locale et supra territoriale } \\
\text { en partenariat avec les } \\
\text { organismes } \\
\text { professionnels agricoles } \\
\text { du département et du } \\
\text { bassin de production }\end{array}$ \\
\hline $\begin{array}{l}\text { Enrayer la } \\
\text { diminution des } \\
\text { surfaces viticoles } \\
\text { approvisionnant } \\
\text { la coopérative } \\
\text { viticole }\end{array}$ & $\begin{array}{l}\text { Portage foncier et } \\
\text { exploitation collective } \\
\text { temporaire }\end{array}$ & $\begin{array}{l}\text { Innovation } \\
\text { technologique et } \\
\text { organisationnelle } \\
\text { Processus } \\
\text { coopératif }\end{array}$ & $\begin{array}{l}\text { Coordination privée par } \\
\text { les viticulteurs } \\
\text { coopérateurs au sein de la } \\
\text { SCEA Dyonis }\end{array}$ \\
\hline
\end{tabular}




\begin{tabular}{|c|c|c|c|}
\hline $\begin{array}{l}\text { Sécuriser la } \\
\text { vocation des } \\
\text { espaces agricoles } \\
\text { et enrayer la } \\
\text { diminution des } \\
\text { surfaces }\end{array}$ & $\begin{array}{l}\text { Mission de développement } \\
\text { économique « agriculture } \\
\text { durable »; grille des prix } \\
\text { du foncier agricole ; veille } \\
\text { et réserve foncière }\end{array}$ & $\begin{array}{l}\text { Innovation sociale } \\
\text { et institutionnelle } \\
\text { Processus } \\
\text { concertatif }\end{array}$ & $\begin{array}{l}\text { Coordination Cdc et } \\
\text { gouvernance publique - } \\
\text { privée associant les } \\
\text { responsables } \\
\text { professionnels oléronais, } \\
\text { la SAFER et les services } \\
\text { de l'État }\end{array}$ \\
\hline $\begin{array}{l}\text { Distinguer les } \\
\text { produits viticoles } \\
\text { oléronais des } \\
\text { autres vignobles } \\
\text { mais aussi, au } \\
\text { sein même des } \\
\text { AOC et IGP } \\
\text { relatives au } \\
\text { bassin de } \\
\text { production du } \\
\text { Cognac }\end{array}$ & $\begin{array}{l}\text { Déclinaison de la mention } \\
\text { « île d’Oléron » dans le } \\
\text { cahier des charges de } \\
\text { l’IGP et utilisation non } \\
\text { légale dans le cadre des } \\
\text { productions relevant de } \\
\text { l’AOC }\end{array}$ & $\begin{array}{l}\text { Innovation sociale } \\
\text { et institutionnelle } \\
\text { Processus } \\
\text { conflictuel }\end{array}$ & $\begin{array}{l}\text { Coordination sectorielle } \\
\text { locale et supra territoriale } \\
\text { en partenariat avec les } \\
\text { organismes } \\
\text { professionnels agricoles } \\
\text { du département et du } \\
\text { bassin de production et } \\
\text { l'INAO }\end{array}$ \\
\hline $\begin{array}{l}\text { Promouvoir } \\
\text { l'identité agricole } \\
\text { et viticole de l'île } \\
\text { d’Oléron et les } \\
\text { savoir-faire } \\
\text { associés }\end{array}$ & $\begin{array}{l}\text { Balades organisées à vélo } \\
\text { « Deux roues pour une } \\
\text { île » } \\
\text { Film Vin'île avec co- } \\
\text { financement de la Cdc }\end{array}$ & $\begin{array}{l}\text { Innovation sociale } \\
\text { et institutionnelle } \\
\text { Processus } \\
\text { concertatif }\end{array}$ & $\begin{array}{l}\text { Coordination par le le } \\
\text { secteur associatif } \\
\text { (GEDAR puis GEDAR et } \\
\text { Marennes Oleron TV) }\end{array}$ \\
\hline $\begin{array}{l}\text { Renforcer la } \\
\text { filière viticole et } \\
\text { promouvoir un } \\
\text { tourisme } \\
\text { patrimonial } \\
\text { alternatif et } \\
\text { complémentaire } \\
\text { au tourisme } \\
\text { balnéaire }\end{array}$ & $\begin{array}{l}\text { Labellisation Vignoble et } \\
\text { Découverte et animation } \\
\text { du dispositif promotionnel } \\
\text { et de mise en réseau des } \\
\text { partenaires }\end{array}$ & $\begin{array}{l}\text { Innovation sociale } \\
\text { et institutionnelle } \\
\text { Processus } \\
\text { concertatif }\end{array}$ & $\begin{array}{l}\text { Coordination Cdc et } \\
\text { gouvernance publique - } \\
\text { privée associant } \\
\text { l'ensemble des } \\
\text { partenaires de la } \\
\text { destination }\end{array}$ \\
\hline
\end{tabular}

596 Tableau 1 : Caractérisation des différentes innovations recensées

Source : Auteurs

$598 \mathrm{Au}$ regard des différentes pressions, la diminution des surfaces en vigne tout au long du $\mathrm{XX}^{\mathrm{e}}$ siècle pouvait laisser présager la disparition du vignoble d’Oléron. Néanmoins, on assiste à une

600 reprise timide des plantations depuis les années 2010. Dès lors, nous avons tenté de mettre en évidence les différents facteurs qui ont conduit à la résistance spatiale, culturelle et économique

602 de la viticulture à Oléron. 
Deux principales stratégies faisant appel à plusieurs innovations, ont permis le maintien de la

604 filière : d'une part, l'adaptation des outils de production aux marchés émergents en lien avec le tourisme et d'autre part la mobilisation en résistance à des projets d'aménagement du territoire 606 et à des politiques de restructuration de la filière. En étroite interaction avec une diversité d'acteurs tels que les collectivités locales, les syndicats, les organismes professionnels agricoles

608 et les organismes de défense et de gestion, les acteurs de la filière viticole oléronaise ont ainsi déployé une série de nouvelles compétences et partenariats. Dans une dynamique 610 d'apprentissage continue, les innovations techniques, organisationnelles, sociales et institutionnelles mobilisées ont permis d'atténuer la dépendance au bassin de production du

612 Cognac, par le passage d’une dépendance subie à une «dépendance créatrice » (Perret, cité par François et al, 2006).

614 La viticulture est de plus en plus considérée comme un atout patrimonial et socio-économique pour le territoire après avoir supporté pendant quelques décennies une relative indifférence de

616 la part des collectivités locales. Cette (re)territorialisation s’organise autour des leviers que sont l'action publique partenariale, la durabilité et la valorisation de l’origine géographique. Elle

618 est inséparable du processus de patrimonialisation de l'activité et des productions à l'oeuvre. Les acteurs de la filière viticole oléronaise ont diversifié leurs circuits de commercialisation en

620 empruntant de nouveaux sentiers de croissance économique basés sur des marchés de proximité géographique et sur la valorisation de la singularité du territoire comme instrument de

622 distinction commerciale. Par ailleurs, la distanciation avec le cœur de l'AOC Cognac se poursuit alors que les surfaces en vignes de la sous-appellation Bois Ordinaires n’ont cessé de

624 diminuer. Aujourd'hui, les îles tirent leur épingle du jeu et les vignobles de Ré et d’Oléron représentent 89 \% des surfaces plantées dans cette sous-appellation.

626

Remerciements

628 Nous tenons à remercier Hélène Bailleul pour ses précieux conseils et Nicolas Pelletier pour la réalisation des cartes.

630

Bibliographie 
632 Barthon C., 2002. Des îles et des ponts, in Brigand L., Les îles de Ponant : histoires et géographie des îles et îlots de la Manche et de l’Atlantique. Édition Palantines, Plomelin, 479 634 p.

Barthon C., 2005. Habiter le patrimoine ou comment gérer les contradictions de la

636 patrimonialisation de l'espace à l'échelle locale : Exemples insulaires de l'atlantique français, in Gravari-Barbas M. (dir), Habiter le patrimoine : Enjeux, approches, vécu, Presses

638 universitaires de Rennes, Rennes, p.351-362.

Bernard G., 1988. Population et vignoble dans les Charentes : un siècle d'évolution originale.

640 Norois, vol. 140, no 1, p. 521-541. DOI : 10.3406/noroi.1988.7505

Bernard G., 1980. La formation des crus de Cognac, Norois, n¹05, p. 89-103. DOI :

642 10.3406/noroi.1980.3873

Bernard G., 2016. Le vignoble des îles charentaises in Coutelle M. (coord.), L'agriculture en

644 Charente Maritime au XXème siècle : guide et jalons pour la recherche, La Crêche, GesteÉditions/Fondation Xavier Bernard, 519 p.

646 Bernard G., 1978. Une production de renommée mondiale en difficulté : le cognac, Norois, n99, p. 431-440. DOI : 10.3406/noroi.1978.3721

648 Bernard G., 1996. La crise du cognac des années 1990, Norois, n¹72, p. 767-774. DOI : 10.3406/noroi.1996.6761

650 Brigand L., 2002. Les îles de Ponant : histoires et géographie des îles et îlots de la Manche et de l'Atlantique. Édition Palantines, Plomelin, 479 p.

652 Brigand L., Chailloux A., Corsi L., David L., Lavialle G., Tesson M., 2019. La marge attractive : les néo-entrepreneurs des îles du Ponant, in Bernier X. (dir), Mobilités et

654 marginalités, Rennes, Collection : Espace et Territoires, Presses Universitaires de Rennes, p.205-218.

656 Chiffoleau Y., 2019. Les circuits courts alimentaires : entre marché et innovation sociale, Édition Eres, Toulouse, Collection : Sociologie économique, 176 p. DOI :

658 10.3917/eres.chiff.2019.01.

Crenn C., Téchoueyres I., 2005. Territoire viticole, patrimoine et distinction : le cas du Pays

660 foyen, Sud-Ouest Européen, vol. 19, n 1, p. 49-55.

Denieau P., 1994. La vigne dans l'île d'Oléron, l'histoire du vignoble, Éditions Local,

662 Collection : Les Cahiers de l'île d’Oléron, n¹6. 23 p.

Dumont R., 1951. Voyages en France d'un agronome, Éditions M.-Th.Génin, Librairie de

664 Médicis, Paris. 467 p. 
Dumortier, B., 1976. Belle-Ile, Houat, Hoëdic : le poids de l'insularité dans trois îles de 666 Bretagne méridionale, Éditions École Normale Supérieure de jeunes filles, Paris, 163 p. GEDAR, Marennes Oléron TV, 2015. Vin'Ile Histoire De La Viticulture oléronaise, du

668 Phylloxera à nos jours. DVD, 41 minutes. https://tube.mo-tv.fr/cat/2015/video/vin-ilehistoire-de-la-viticulture-oléronaise-du-phylloxera-a-nos-jours

670 Guillemet D., 1999. Les paysanneries insulaires du Ponant (XVIIe XIXe siècles) : typologie, stratigraphies et évolutions, in Antoine A. (dir), Campagnes de l'Ouest : Stratigraphie et

672 relations sociales dans l'histoire, Rennes, Presses Universitaires de Rennes, p 443-464. Guillemet D., 2000. Les îles de l'Ouest de Bréhat à Oléron, du Moyen âge à la Révolution,

674 Geste Édition, La Crèche, Collection : Pays d'histoire, 355 p.

Hervieu B., Viard J., 2005. Au bonheur des campagnes : et des provinces, La Tour-d'Aigues,

676 Éditions de l'Aube, 154 p.

Lebahy Y., Le Délézir R., 2007. Le littoral agressé : pour une politique volontariste de

678 l'aménagement en Bretagne, Édition Apogée, 190 p.

Maby J., 2002. Paysage et imaginaire : l'exploitation de nouvelles valeurs ajoutées dans les

680 terroirs viticoles, Annales de géographie, n624, vol. 111, p. 198-211. DOI :

10.3406/geo.2002.1666

682 Nadreau G., 1994. La vigne et sa conduite, in La vigne dans l'île d'Oléron, la main de l’homme, Éditions Local, Collection : Les Cahiers d’Oléron, nº17.

684 Pecqueur B., 2014. Esquisse d’une géographie économique territoriale, L’Espace géographique, n ${ }^{\circ}$ 3, vol. 43 p. 198-214.

686 Pecqueur B., Peyrache-Gadeau V., 2010. Fondements interdisciplinaires et systémiques de l'approche territoriale : Introduction, Revue d’Économie Régionale Urbaine, n4, p. 613-23.

688 Péres S., 2009. La résistance des espaces viticoles à l'extension urbaine, le cas du vignoble de Bordeaux, Revue d’Économie Régionale Urbaine, n¹, p. 155-177. DOI :

690 10.3917/reru.091.0155.

Péron F., 1993. Des îles et des hommes : l'insularité aujourd'hui. Éditions de la Cité, 286 p.

692 Rieutort L., 2009. Dynamiques rurales françaises et re-territorialisation de l'agriculture, L'Information géographique, $\mathrm{n}^{\circ} 1$, vol. 73, p. 30-48. DOI :10.3917/lig.731.0030

694 Sanson B., 2008. Comment appréhender l'espace et les activités agricoles littorales pour assurer leur maintien durable? Étude à partir du cas de l'île d'Oléron. Mémoire de master 2, 696 Agrocampus Ouest Rennes, 79 p. 
Torre A., 2015. Théorie du développement territorial, Géographie, économie, société, n³, vol. 17, p. 273-288.

Torre A., 2018. Développement territorial et relations de proximité, Revue d'Économie

698 Régionale \& Urbaine, $\mathrm{n}^{\circ} .5$, p. 1043-1075.

François H., Hirczak M., Senil N., 2006. Territoire et patrimoine : la co-construction d'une

700 dynamique et de ses ressources, Revue d'Économie Régionale \& Urbaine, n5, p. 683-700.

DOI : 10.3917/reru.065.0683.

702 Torre A., Kirat T., Melot R., et al. 2016. Les conflits d'usage et de voisinage de l'espace.

Bilan d'un programme de recherche pluridisciplinaire, L'information géographique, vol. 80,

$704 n^{\circ} 4$, p. 8-29.

Yengué J.-L., Robert A., 2018. Le vignoble périurbain : entre préoccupations locales et

706 contexte global. Exemple de Vouvray (France), Norois, n² 249, p. 21-42.

708 Table des illustrations

Figure 1 : Les étendues en vigne par canton en 1906-1910

710 Figure 1 : Vineyard areas by canton in 1906-1910

Figure 2 : Evolution de la surface viticole dans le bassin de production du Cognac

712 Figure 2 : Vineyard areas progress in Cognac delimited area of production

Figure 3 : Évolution de la viticulture oléronaise depuis le $\mathrm{XIX}^{\mathrm{e}}$ siècle

714 Figure 3 : Oleron Vineyard history since $19^{\text {th }}$

Figure 4 : Les sous-appellations de l’AOC Cognac

716 Figure 4 : Appellations within the Cognac delimited area of production

Figure 5 : Volumes de Cognac commercialisés sur les marchés étrangers et le marché français

718 depuis 1945

Figure 5 : Cognac markets since 1945

720 Figure 6 : Evolution des superficies en vigne par canton de 1976 à 1987

Figure 6 : Decrease vineyard areas by canton in 1976-1987

722 Figure 7 : Evolution des surfaces en vigne et de la démographie viticole depuis 1955 à Oléron 
Figure 7 : Vineyard areas and population progress in Oleron since 1955

724 


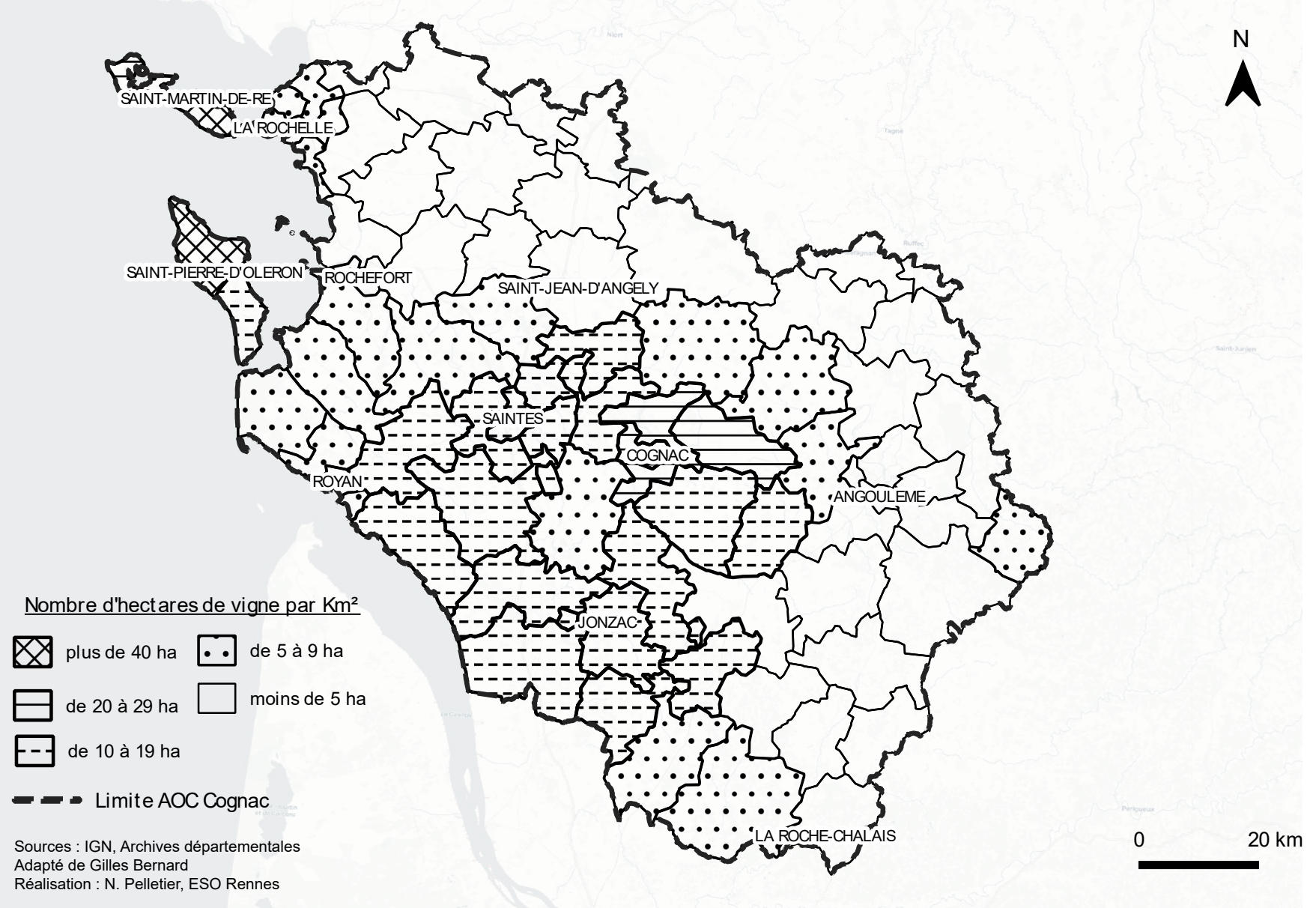




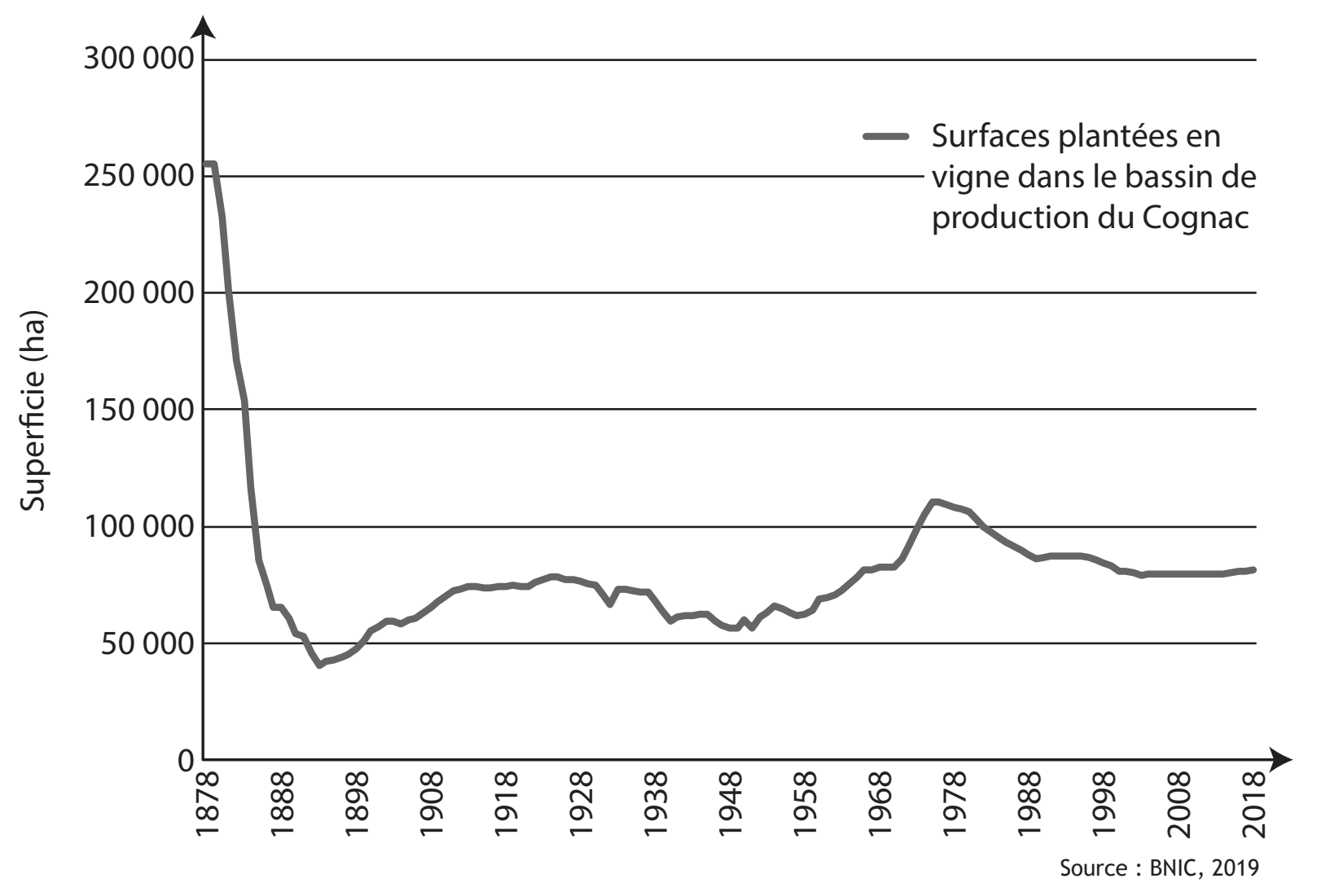

Fig 2 
Evolutions des structures, du fonctionnement et du rôle de la viticulture oléronaise

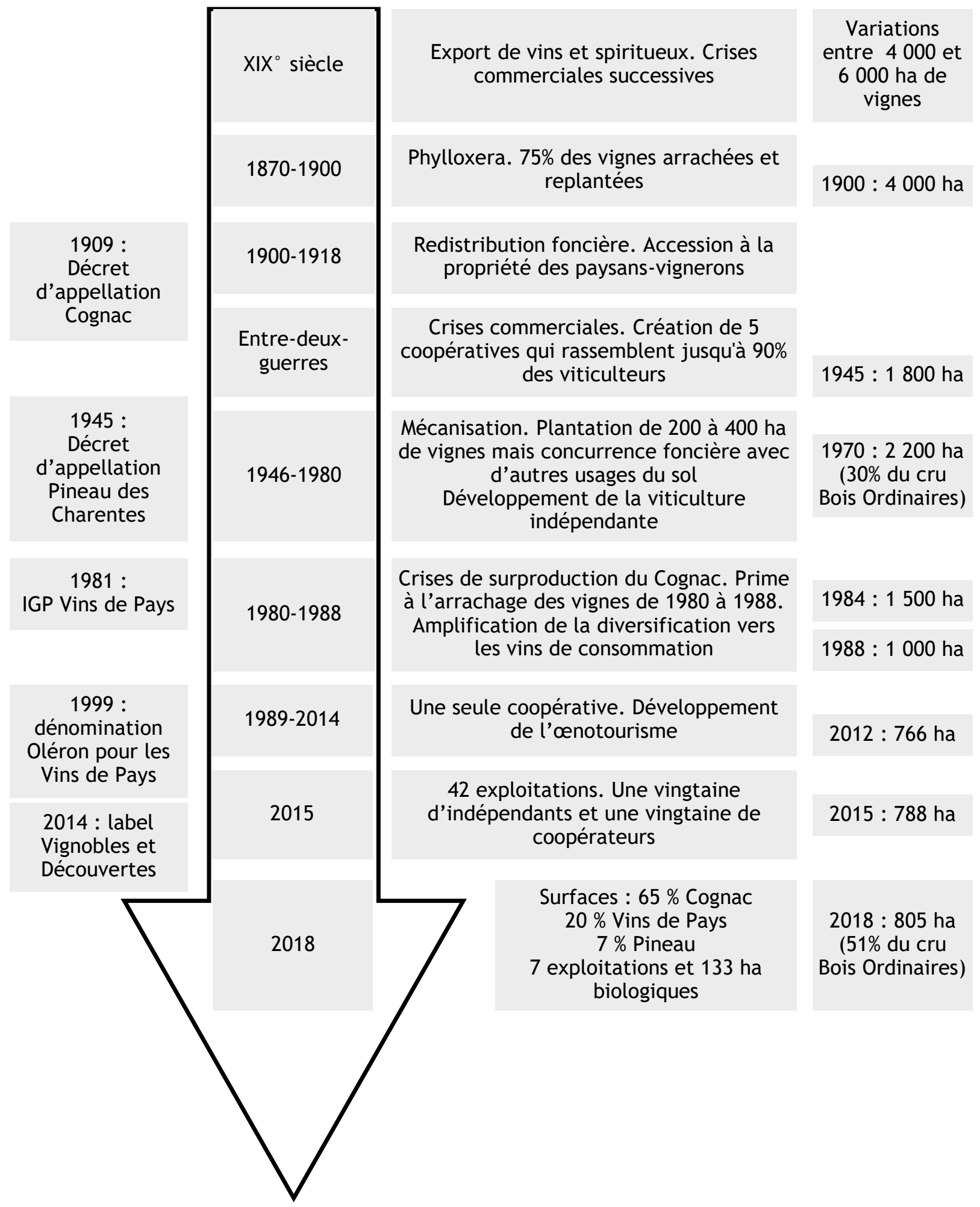


700000

600000

⿳亠丷厂 500000

음

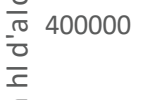

ธ 300000

ฆ

긍 200000

100000

0

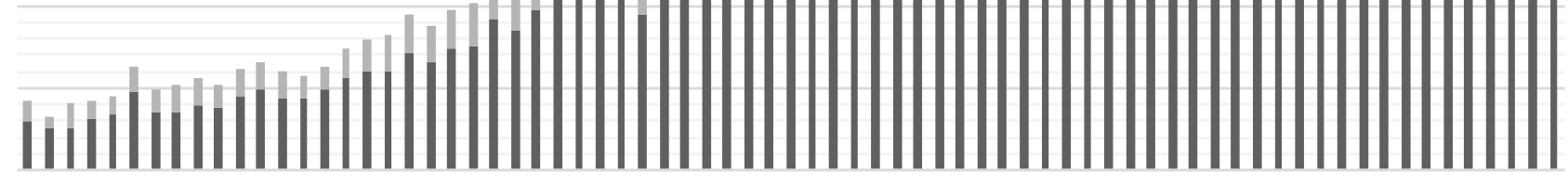

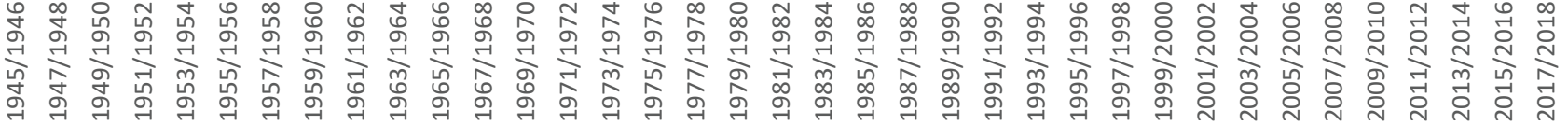

Fig 5 


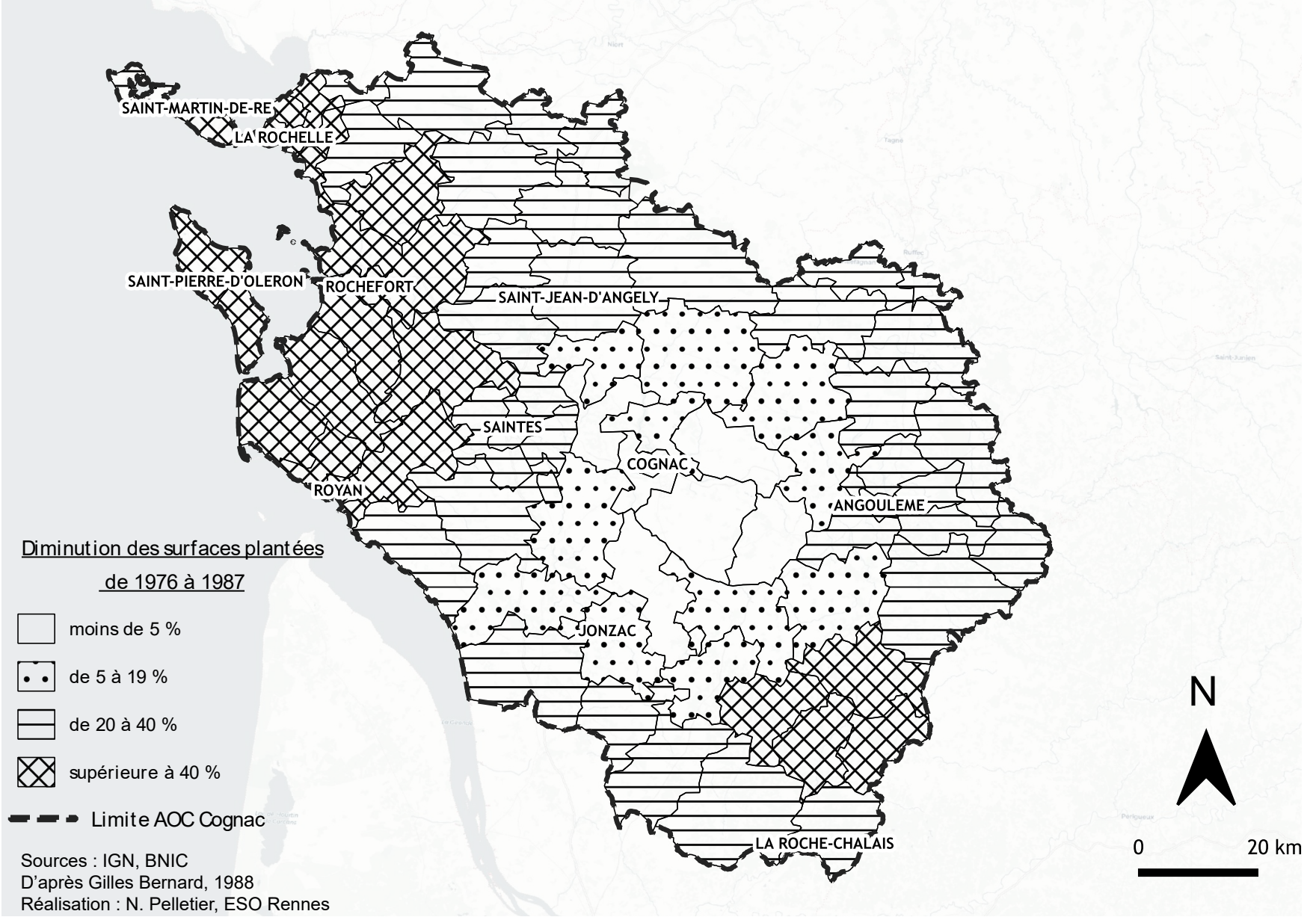


Fig 7

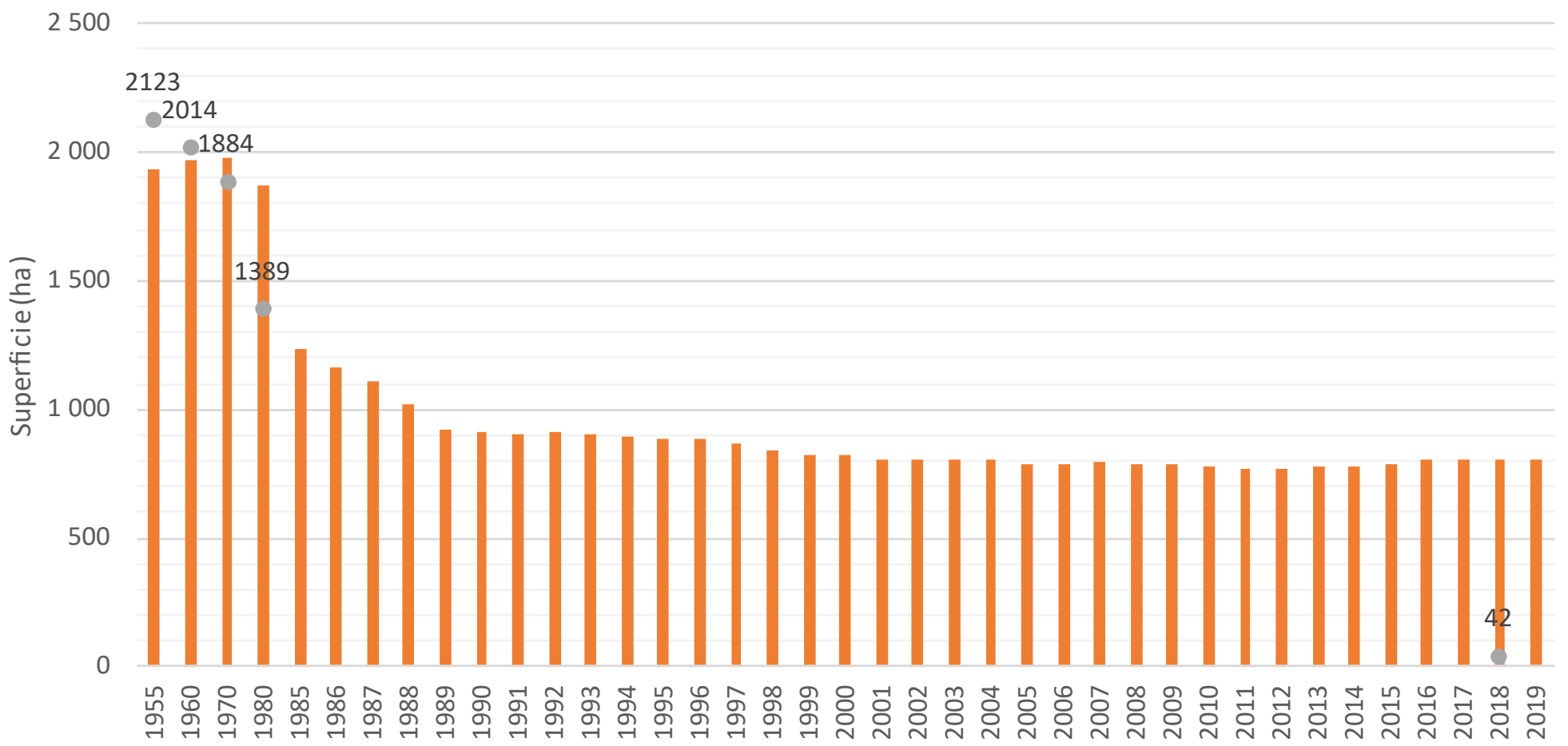

